\title{
Bilateral Cavernous Sinus Thrombophlebitis Caused by MRSA - A Rare Clinical Entity
}

\author{
Article by, Vaishali Sangole ${ }^{1} \&$ Padma Ramesh $^{2}$ \\ ${ }^{1}$ Associate Professor, MGM Medical College, Navi Mumbai \\ Email:vssangole@rediffmail.com \\ Associate Professor, MGM Medical College, Navi Mumbai \\ Email: padmar137@yahoo.com
}

\begin{abstract}
We present a young female who developed bilateral cavernous sinus thrombophlebitis after a week's history of a single acne-like lesion or furuncle over the right lateral ala of the nose which she had squeezed. She was managed aggressively with intravenous antibiotics, heparin and a sliding scale of insulin. Signs and symptoms improved after 2 weeks of treatment, and she was discharged from hospital on an oral anticoagulant.
\end{abstract}

Keywords: Bilateral Cavenous Sinus Thrombosis, Proptosis, Ophthalmoplegia, Nasal Furunculosis, Facial cellulitis

\section{Introduction}

Nasal vestibular furunculosis is a commonly encountered disorder in the general population, characterized by acute localized infection in relation to a hair follicle in the skin lining of the nasal vestibule. Many of these patients are chronic carriers of Staphylococcus aureus (Staph. aureus) in the nasal vestibule. Methicillin-resistant Staphylococcus aureus (MRSA) is a growing threat in hospitals but it is also being reported in the community [1]. Community-acquired MRSA is becoming an important public health problem. New strains of Staph. aureus displaying unique combinations of virulence factors and resistance traits have been associated with high morbidity and mortality. Outbreaks of epidemic furunculosis and cases of severe invasive pulmonary infections in young, otherwise healthy people have been particularly noteworthy.

The habit of nose picking in such circumstances can lead to nasal furunculosis. Uncontrolled diabetes mellitus should be considered as a cause in any patients with nasal septal abscess when trauma is excluded.

Cavernous sinus thrombosis (CST) is a serious life threatening condition with high mortality which has improved markedly because of an early awareness of the condition and prompt initiation of parenteral antibiotics [2]. In our patient, the disease was initially unilateral and then spread through inter-cavernous communications to the contralateral cavernous sinus.

\section{Case report}

A 22 year old female patient was brought to the emergency services of MGM Medical College and Hospital, Kamothe, Navi Mumbai in a drowsy and disoriented state with the chief complaint of noticing a painful swelling on the right ala of the nose for the past 4 days. The swelling was initially pea-sized and non-progressive until she squeezed out about $2 \mathrm{ml}$ of purulent, non-offensive non-sanguinous discharge from it. The next day, the swelling had extended to the right lateral dorsum and tip of the nose, right periorbital region and eyelids. The swelling progressed to involve the left eye over the next 24 hours and increased in the next 48 hours to an extent that the patient found it difficult to open both her eyes. There was an associated high grade intermittent fever from onset. The patient was unresponsive to questions and history was obtained from the accompanying relative. There was no history of nasal picking, URTI, nasal allergies, dental infection, recurrent furunculosis, convulsions, features of cranial nerve involvement, diabetes mellitus or immunocompromised state.. 
South American Journal of Clinical Research

Volume 3, Issue 1, 2016

On examination, the patient was stuporous and not responding to verbal commands (Glasgow Coma Scale 5/15, E1M4V0). She was febrile $102^{0} \mathrm{~F}$, with tachycardia 150/min, BP of 128/80 mmHg, tachypneic 34/min and a SPO2 of $96 \%$ on room air. Local examination showed a draining abscess over the right nasal ala with evidence of cellulitis over the right ala and nasal dorsum and adjacent right cheek. On palpation over the right nasal ala, about $2 \mathrm{ml}$ of frank pus extruded freely through two discharging points. The next day, the swelling had progressed to a diffuse swelling over nose (more on the right side) with crusting. Anterior rhinos copy showed dry hard crusts occluding the right nasal vestibule which were removed, but was otherwise normal. There was bilateral frontal and ethmoid sinus tenderness and pitting oedema over the forehead. There was bilateral axial proptosis of $21 \mathrm{~mm}$ (right) and 22 mm (left) with bilateral severe periorbital oedema, mucopurulent conjunctival discharge, bilateral severe chemosis and conjunctival congestion with lid margins bleeding on attempted separation [Figure 1]. Pupils were $3 \mathrm{~mm}$, round, regular, and reacting to light. Fundoscopy was normal. Visual acuity and eye movements could not be assessed at presentation due to her unresponsive state and inability to open eyes.

Investigation revealed anaemia with haemoglobin of $9.4 \mathrm{~g} / \mathrm{dL}$ on admission which declined to $6.6 \mathrm{~g} / \mathrm{dL}$ over the next four days. TLC was $16060 / \mathrm{mm}^{3}$ and she was seronegative for HIV, hepatitis B and hepatitis C. Random blood sugar was $120 \mathrm{mg} / \mathrm{dL}$ on admission but levels ranged between $165-254 \mathrm{mg} / \mathrm{dL}$ on the next day. HbA1C was $5.2 \%$. Platelet count was $156000 / \mathrm{mm}^{3}$ and PT and INR were $15.1 \mathrm{sec}$ and 1.09 respectively.

Pus culture from the abscess showed MRSA which was sensitive to levofloxacin, cefotaxime and gentamicin, with intermediate sensitivity to tetracycline. Blood culture was sterile after 72 hrs of incubation. Nasal scraping examined on a $\mathrm{KOH}$ mount was negative for fungus.

MRI scan with contrast venography of brain and orbits showed both cavernous sinuses thrombosed and thickened in T1W images with no contrast filling the sinuses [Figure.2]. Bilateral superior ophthalmic veins were thrombosed and enlarged. Cavernous portions of both internal carotid arteries were patent but showed mild narrowing. Cellulitis was noted involving subcutaneous regions of nose on the right side and conal and extraconal compartments of both orbits. There were no radiological signs of a cerebral abscess.

She was initially placed on intravenous antibiotics: piperacillin-tazobactam $4.5 \mathrm{gm} \mathrm{BD}$, amikacin $500 \mathrm{mg}$ IV BD, metronidazole $500 \mathrm{mg}$ TID along with subcutaneous fractional Low Molecular Weight Heparin (Enoxaparin) $40 \mathrm{mg}$ OD, Tab Acenocoumarol $3 \mathrm{mg}$ OD, and soluble aspirin $150 \mathrm{mg}$ OD. Following evidence of hyperglycaemia on the second day, a sliding scale of Human Actrapid insulin infusion was begun which was followed by a titrating scale for the next 5 days.

Adjunctive treatment included Tab Phenytoin sodium $100 \mathrm{mg}$ OD through nasogastric tube, Moxifloxacin eye drops and Calcium chloride eye ointment. After the pus culture and sensitivity report was received, the antibiotic regime was changed to intravenous Levofloxacin $500 \mathrm{mg}$ OD, Targocid $400 \mathrm{mg}$ OD, and Meropenem 1 gm TID.

Her clinical status showed a progressive steady improvement with improvement in local and periorbital swelling, eyelid oedema and proptosis (reducing to $16 \mathrm{~mm}$ and $18 \mathrm{~mm}$ for right and left eyes respectively) and a visual acuity of finger counting at 3 metres by the fifth day. She was a febrile by the fifth day. Blood sugar levels ranged between 111-420 mg/dL from the second to fifth days following which they remained normal $(82-188 \mathrm{mg} / \mathrm{dL})$ and thereafter she was managed without insulin on a diabetic diet. Following a single blood transfusion, haemoglobin level came up to $7.7 \mathrm{~g} / \mathrm{dL}$ and was $8.8 \mathrm{~g} / \mathrm{dL}$ at discharge from hospital. TLC progressively declined to $6300 / \mathrm{mm}^{3}$ at discharge. She was discharged to home after a month on a diabetic diet and oral anticoagulant.

\section{Discussion}

Nasal furunculosis is a quite common clinical entity during pre-monsoon and monsoon period as humidity level is very high (more than 90\%) during this period. A surge of cases 
with complications are seen in the dry winter months. Because of the dry cold air that is inhaled there is increased crust formation. Plucking of nasal hair with a nasal trimmer may also lead to nasal furunculosis.

The causative organism is almost always Staph. aureus. The source of staphylococcal infection is usually the nose or the perineum and it is thought that the infection is disseminated by fingers and clothing.

Diabetes mellitus is the most common predisposing factor associated with complications. Other conditions associated with furunculosis include obesity [3] and immune compromise as with HIV infection, blood dyscrasias and treatment with immunosuppressive drugs. A neglected nasal furuncle, paranasal sinusitis or allergic rhinitis can lead to CST $[4,5,6]$

CST is the formation of thrombus within the cavernous sinus, which can be either septic or aseptic. Septic CST is a rapidly evolving thrombophlebitic process with an infectious origin typically from the middle third of face (sinuses, ears, teeth, or mouth), affecting the cavernous sinus and its structures. Aseptic CST is usually a thrombotic process that is a result of trauma, iatrogenic injuries, or prothrombotic conditions [7]. CST is usually a late complication of an infection of the central face and has a fulminant course with high mortality and morbidity. The diagnosis of septic CST requires a high index of suspicion. Confirmation by imaging, early diagnosis and surgical drainage of the underlying primary source of infection in conjunction with long-term intravenous antibiotic therapy is critical for an optimal clinical outcome [8].

The danger triangle of the face consists of the area from the corners of the mouth to the bridge of the nose, including the nose and maxilla $[9,10]$. Due to the special nature of the blood supply to the human nose and the surrounding area, it is possible for retrograde infections from the nose area to spread to the brain causing cavernous sinus thrombosis, meningitis or brain abscess. Most people, but not all, have valves in the veins of the face [11].

The cavernous sinuses lie within the cranial cavity, between the layers of meninges and are a major conduit of venous drainage from the brain [12]. They are two irregular shaped, endothelium lined venous spaces, on either side of sphenoid bone with wide connections to the veins draining the face. They are so named because they present a reticulate structure, which is traversed by numerous interlacing filaments.

The cavernous sinuses receive venous blood from the facial veins (via the superior and inferior ophthalmic veins) as well as the sphenoid and middle cerebral veins. They, in turn, empty ultimately into the internal jugular veins and sigmoid sinuses via the superior petrosal sinuses. This complex web of veins is valueless; blood can flow in any direction. Infections of the face especially in danger area zone $[9,10]$ (as in our case) can spread to this vein complex.

The sinus contains the internal carotid artery, which passes along the medial wall, and the occulomotor, the trochlear, the ophthalmic and maxillary or divisions of the trigeminal nerve which pass along the lateral wall. Just below the artery is the abducent nerve.

The facial vein is connected with the cavernous sinus by many anastomoses; angular vein, supra-orbital and infra-orbital veins, the superior and inferior ophthalmic veins, the nasal veins, the facial communicating veins, and the pterygoid vein, all of which either directly or indirectly communicate with the cavernous sinus.

Patients with CST present with fever, headache, vomiting, proptosis and involvement of 3rd, 4th and 6th cranial nerves. Cavernous sinus thrombosis is usually ushered in by a headache and initial chills. There is a high fever from the start, ranging from $102^{0} \mathrm{~F}-106^{0} \mathrm{~F}$. When the clot begins to soften and break down, the temperature fluctuates. Nausea and vomiting come on early and may be of projectile type. Besides the symptoms of general sepsis, the cardinal diagnostic signs are produced by venous obstruction and pressure on adjacent nerves. The former causes exophthalmos, proptosis, oedema of the lids, chemosis of the conjunctiva; the latter, headache and eye pain due to irritation of the fifth cranial nerve, paralysis of the eye muscles and rigidity and dilatation of the pupil from paralysis of the third, fourth and sixth cranial nerves and impairement of vision and choked disc due to involvement 
South American Journal of Clinical Research

Volume 3, Issue 1, 2016

of optic nerve. The second eye is almost always affected within 48 hours after the first, this being the most important sign distinguishing CST from orbital cellulitis.

Leucocytosis in CSF is marked (except when the patients resistance is low), with increased polymorphonuclears ranging from $85-98 \%$, while the haemoglobin and red blood cells are decreased.

Culture and sensitivity are indicated. In septicemia, the blood culture will reveal the etiologic organisms. In complicated septic meningitis, which is indicated by positive Kernig and Brudzinski signs and opisthotonus, the spinal fluid is cloudy, with increased pressure, many cells per field (the majority being polymorphonuclear leucocytes), increased globulin and decreased sugar levels.

CT and MRI scans establish the diagnosis.

The differential diagnosis of CST includes orbital cellulitis, superior orbital fissure syndrome, orbital apex syndrome, sino-orbital aspergillosis, subperiosteal mucocoeles, Tolosa-Hunt's syndrome, meningioma, carotid-cavernous fistula, and rhino-cerebral mucormycosis. The clinical presentation is usually due to the venous obstruction (either ophthalmic veins or others) as well as impairment of the cranial nerves at cavernous sinus wall or within the trabecula.

The course of cavernous sinus thrombosis varies from 2 to 18 days. Death occurs mostly within a week after development of eye symptoms and is due to meningitis. While the disorder has become less common with antibiotics, it still carries a high risk of death and needs to be treated aggressively with antibiotics and blood thinners [13].

An internet search revealed very few reported cases of bilateral CST. A case of bilateral ophthalmic vein thrombosis secondary to nasal furunculosis was reported by Rohana in 2009; however, this teenage female patient had only unilateral CST [14]. Komatsu et al (2013) reported an unusual case of unilateral CST caused by contralateral sphenoid sinusitis; in this case, there was no evidence of CST on the affected side [15].

Only 2 cases of bilateral CST have been reported prior to our case. Rajesh. V et al in 2013 reported bilateral CST and facial palsy as complications of a dental abscess in a 50-year-old, previously healthy, female patient. Pus from the abscess grew Staph. aureus [16]. In 2006, Abound et al reported bilateral CST complicating sinusitis in a previously healthy, 6-year-old boy who presented with headache for one week. Although clinically this patient had only unilateral periorbital redness and swelling, CT scan revealed bilateral cavernous sinus and left orbital vein thrombosis, as well as sphenoid and ethmoidal sinusitis and a left preseptal cellulitis. Blood culture grew Streptococcus milleri [17]. All three patients with bilateral CST including our patient, made a complete recovery. In our case, diabetes mellitus which was detected only after admission to hospital, was a predisposing factor.

\section{Conclusion}

This case of bilateral cavernous sinus thrombosis which presented with a full-blown clinical picture developed secondary to nasal furunculosis and abscess caused by MRSA in a background of hyperglycaemia. Prompt intervention with intravenous antibiotics, insulin and anticoagulants resulted in a good patient outcome. Health education regarding prompt and correct management of infections over the dangerous area of the face will help to reduce the incidence of this fulminant complication.

\section{References}

[1.] Absoud M, Hikmet F, Dey P, Joffe M, Thambapillai E, 'Bilateral cavernous sinus thrombosis complicating sinusitis’. J R Soc Med. (2006), 99, pp.474-476.

[2.] Baidya K. Bhaduri G, Mondal LK, 'Atypical presentation of a unilateral septic thrombosis of cavernous sinus'. J. Indian Med Association. (2005), 103, pp.437-8.

[3.] Canon ML, Antonio BL, McCloskey JJ, 'Cavernous sinus thrombosis complicating sinusitis'. Paiatr Crit Care Med. (2004), 5(1), pp.86-8. 
[4.] Chacar-Rabay H, Hejeily RK, Aouad A, 'Cavernous sinus thrombosis. Late diagnosis and complications’. JM ed Liban. (1998), 4, 6pp.218-21.

[5.] Hollinshead WH, ‘Anatomy of Surgeons', The Head and Neck. New York, USA: Harper \& Row. (1968).

[6.] Hom. Milton M, Bielory Leonard, 'The anatomical and functional relationship between allergic conjunctivitis and allergic rhinitis. Allergy and Rhinology’. (2013), 4(3), pp.110-119.

[7.] Komatsu H, Matsumoto F, Kasai M, Kurano K, Sasaki D and Ikeda K, 'Cavernous sinus thrombosis caused by contralateral sphenoid sinusitis: a case report’. Head \& Face Medicine. ( 2013), pp. 9-9.

[8.] Okamoto H, Ogata A, Kosugi M, 'Cavernous sinsus thrombophlebitis related to dental infectiontwo case reports'. Neurol Med Chir. (2012), 52(10), pp.757-60.

[9.] Osborn AG, ‘Craniofacial venous plexuses: Angiographic Study’. AJR. (1981), 136, pp.139-143.

[10.] Radulescu L, Poita I, Curca I A, 'Thrombophlebitis of left cavernous sinus secondary to acute right sphenoid sinusitis’. Rev Med Chir Soc Med Nat lasi. (2005), 109, pp.573-6.

[11.] Rajesh V, Vivek J, Rakesh KS, Hari R, and Uma SPl, 'Bilateral cavernous sinus thrombosis and facial palsy as complications of dental abscess’. Natl J Maxillofac Surg. (2013), 4(2), pp. 252-255.

[12.] Rohana AR, Rosli MK, Nik Rizal NY, Shatriah I and Wan Hazabbah WH, 'Bilateral Ophthalmic Vein Thrombosis Secondary to Nasal Furunculosis’. Orbit. (2008), 27, pp.215-217.

[13.] Scheinfeld NS, 'Obesity and dermatology’. Clin Dermatol. (2004)), 22(4), pp.303-9.

[14.] Sofferman RA, 'Cavernous sinus thrombophlebitis secondary to sphenoid sinusitis'. Laryngosope. (1983), 93, pp.797-800.

[15.] Yarington CT, ‘Cavernous sinus thrombosis revisited’. Proc R Soc med. (1977), 70, pp.456-459.

[16.] Zetola N, Francis JS, Nuermberger EL, 'Community-acquired meticillin-resistant Staphylococcus aureus: an emerging threat’. Lancet Infect Dis. (2005), 5(5), pp.275-86.

[17.] Zhang J, Stringer MD, 'Opthalmic and facial veins are not valveless'. Clin Experiment Ophthalmology. (2010), 38(5), pp.502-10.

\section{Figures}

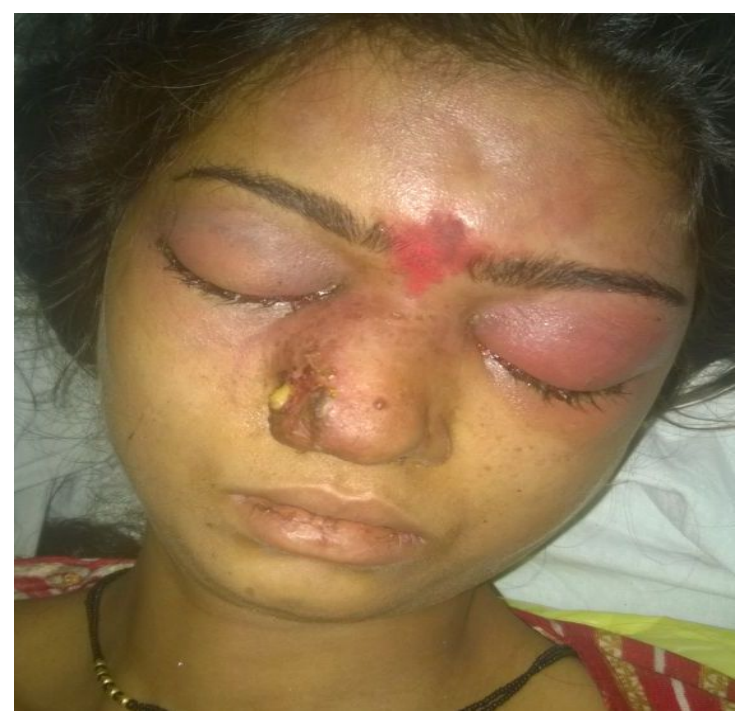

Figure 1. A 22 year old female in stuporous state with a pustular lesion over right nasal ala, bilateral periorbital oedema and proptosis. 
South American Journal of Clinical Research

Volume 3, Issue 1, 2016

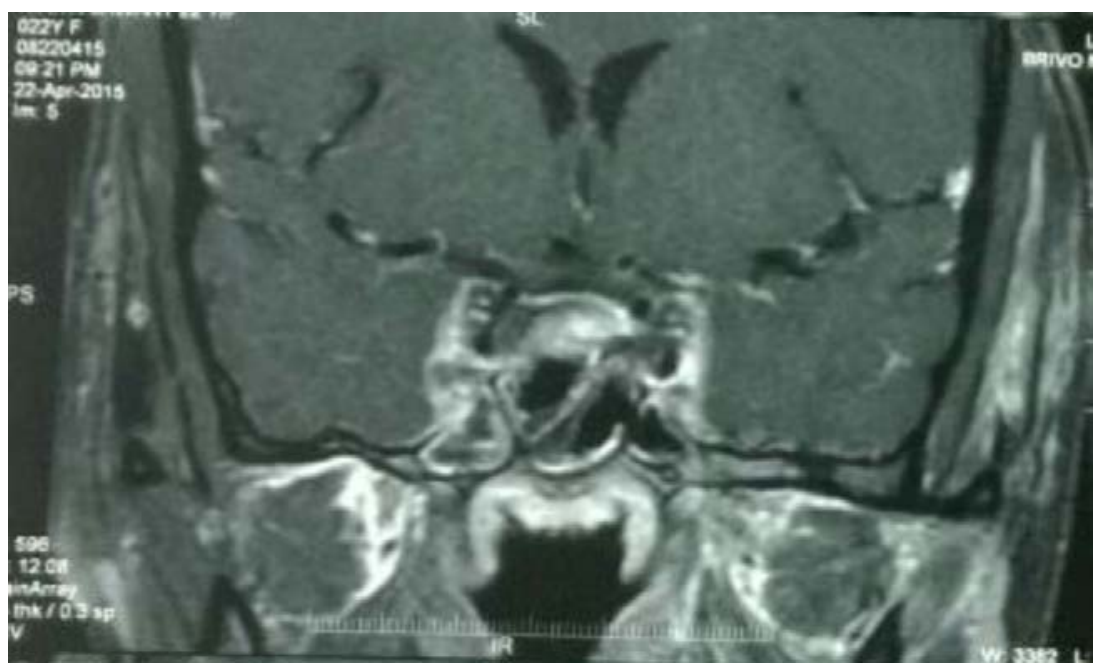

Figure 2. Coronal MRI T1 W image, post contrast showing bulky appearance of the right cavernous sinus with an abnormal lateral bulge and heterogenous filling defects. Findings are similar but milder on left.

\section{Author's profile.}

\section{Dr. Vaishali Sangole}

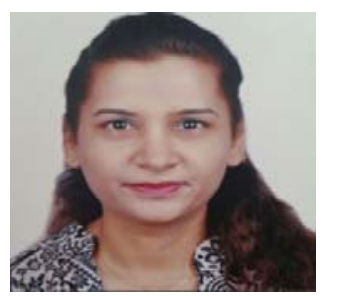

Medical Officer: Gram Seva Trust, Kharel, Gujarat. 23-6-97 to 22-6-99.

Residency (ENT): Mahatma Gandhi Institute of Medical Sciences, Sevagram. 1-9-1999 to 31-3-2002.

Lecturer ( ENT): Mahatma Gandhi Institute of Medical Sciences, Sevagram.17-08-2002 to 6-05-2004

Clinical Associate (ENT): P.D. Hinduja National hospital \& Research centre, Mumbai.3010-2005 to 31-03-2008

Astt. Professor (Lecturer) (ENT): MGM Hospital and Medical College, Kamothe, Navi Mumbai.

1-04-2008 to 31.7.2011

Associate Professor (ENT): MGM Hospital and Medical College, Kamothe, Navi Mumbai. 1.8.2011 till date 


\section{Dr. Padma Ramesh}

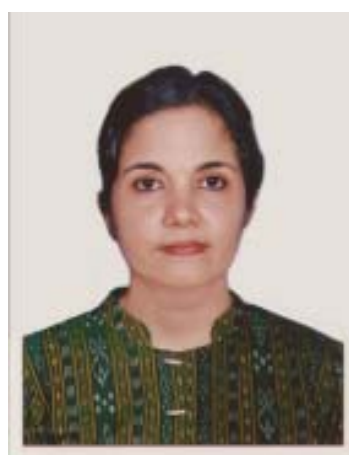

A medical graduate of Government Medical College, Trivandrum, Kerala, India, Dr Padma Ramesh completed her MS (ENT) from the Armed Forces Medical College, Pune in 2000. After a professional career of 22 years in the Armed Forces, she joined MGM Hospital and Medical College, Kamothe, Navi Mumbai in 2013 where she is currently Associate Professor (ENT). 